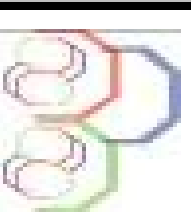

Journal of Applied Biosciences 97:9152 - 9162

ISSN 1997-5902

\title{
Évaluation des propriétés antimicrobiennes des javels vendues à Lomé sur quelques germes isolés de l'eau de consommation
}

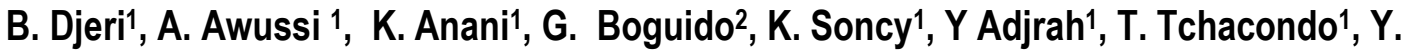 \\ Ameyapoh 1, L.M. Bawa² et DS Karou'1. \\ 1 : Laboratoire de Microbiologie et de Contrôle de Qualité des Denrées Alimentaires, Ecole Supérieure des \\ Techniques Biologiques et alimentaires (ESTBA), Université de Lomé, Togo \\ 2 : Laboratoire de Chimie des Eaux, Facultés des Sciences (FDS) Université de Lomé BP 1515, Lomé Togo \\ Corresponding author: Karou D. Simplice simplicekarou@hotmail.com
}

Original submitted in on 22 no November 2015. Published online at www.m.elewa.org on $31^{\text {st }}$ January 2016 http://dx.doi.org/10.4314/jab.v97i1.2

\section{RÉSUMÉ}

Objectif : II s'est agi de l'évaluation de l'efficacité de différentes marques d'eau de javel vendues à Lomé sur quelques germes isolés des eaux de consommation.

Matériel et méthodes : Cette étude a consisté en une analyse microbiologique de six échantillons d'eau de consommation et en une identification des germes présents en utilisant les normes AFNOR. Quatre germes isolés de ces eaux et un germe de référence (Escherichia coli CIP 105 182) ont été ensuite testés avec des échantillons de javel. II s'agit des streptocoques fécaux, des coliformes totaux, de Escherichia coli et des Bacilles à Gram positif. La technique de dilution en milieu liquide avec le bouillon Muller Hinton a été utilisée pour ces tests. Les degrés chlorométriques de ces eaux de javel ont été ensuite déterminés par iodométrie.

Résultats: L'analyse microbiologique des échantillons d'eau de consommation a montré qu'ils étaient de qualité hygiénique non satisfaisante par rapport à la flore aérobie mésophile totale, aux coliformes totaux et thermotolérents et aux streptococoques fécaux. Toutes les marques d'eau de Javel ont inhibé à des degrés divers la croissance de tous les germes testés. Ainsi, les CMB des eaux de Javel 5, Javel 4, javel 3 testées sur les germes totaux ont été de 1,66\% (v/v) alors que celles des eaux de Javel 6 et 7 ont été respectivement de $8,33 \%$ et de $6,25 \%$ (v/v) pour un temps réactionnel de 3 à 6 heures. En ce qui concerne les degrés chlorométriques, toutes les eaux de javel testées avaient des degrés chlorométriques inférieurs à ceux mentionnés sur les emballages. Conclusion: Cette étude a montré qu'une analyse continue des eaux de javel vendues au Togo est indispensable pour s'assurer de l'efficacité des traitements de désinfection par ces javels.

Mots clés : bactéries, eau, javel, désinfection, antimicrobiens, Togo 
Evaluation of the antimicrobial properties of bleaches sold in Lomé on some bacteria isolated from drinking water

Objective: This study aimed to assess the effectiveness of different bleach sold in Lomé on some bacteria isolated from drinking water.

Materials and Methods: This consisted of microbiological analysis of six samples of consumption water and identification of germs using AFNOR standards methods. Four germs isolated from these waters and a reference strain (Escherichia coli CIP 105182) was then tested with samples of bleach. The chlorometric degrees of these bleaches were also determined using iodine method.

Results: Microbiological analysis of consumption water samples showed that these samples were of unsatisfactory hygienic quality according to mesospheric germs, coliforms and fecal streptococci. All bleaches used inhibited the growth of all tested bacteria. Thus the MBC of bleaches 5, 4 and 3 tested on total aerobic flora were equal to $1.66 \%(\mathrm{v} / \mathrm{v})$ while those of bleaches 6 and 7 were $8.33 \%$ and $6.25 \%(\mathrm{v} / \mathrm{v})$, respectively. The reaction time varied from 3 to 6 hours. Concerning the chlorometric degrees, all bleaches tested had chlorometric degrees lower than those mentioned on the packaging.

Conclusion: This study showed that the periodic analysis of bleaches sold in Togo is essential to ensure the effectiveness of the disinfection treatments of with these bleaches.

Key words: bacteria, water, bleach, disinfection, Togo

\section{INTRODUCTION}

L'eau constitue le besoin nutritionnel le plus éminent de l'organisme, le corps humain étant constitué à environ $65 \%$ d'eau. L'eau peut également être la source de plusieurs maladies qui entraînent la mort dans certains cas comme le choléra et les autres maladies diarrhéiques (Tessier, 1992 ; Matthys et al., 2006 ; Poda, 2007). C'est la raison pour laquelle la qualité de l'eau de boisson constitue un élément fondamental de la prévention primaire des maladies à transmission hydrique (Lesne, 1998 ; Degbey et al., 2011 ). L'accessibilité à l'eau potable est un problème important auquel se heurtent les populations dans les pays en développement. En l'an 2000, I'OMS a estimé que $40 \%$ des africains n'avaient pas accès à l'eau potable et aux équipements adéquats d'assainissement. Face à cette situation, les populations qui s'approvisionnent en eau de surface, essaient de les traiter à leur manière. Plusieurs méthodes de traitement traditionnel de l'eau sont préconisées, cependant la plus utilisée est le traitement au chlore sous forme d'eau de javel (Bawa et al., 2008). Le Togo à l'instar des autres pays en développement, se trouve confronté au même problème de l'accessibilité à l'eau potable surtout dans les zones rurales. De nos jours on dispose de très peu de données sur la qualité des eaux de consommation dans le pays. Cependant quelques travaux effectués ont montré que les eaux de boisson fournies par le réseau de distribution urbaine ne sont pas toujours de qualité hygiénique satisfaisante (Kimassoum et al., 2011). Il en est de même pour les eaux de forage et de puits, principales sources d'approvisionnement des populations qui ne sont pas incluent dans le réseau de distribution et des populations en zone rurale et semi-urbaine (Tampo et al., 2014). Pour ces dernières la désinfection des eaux est faite de façon empirique à l'aide l'eau de javel vendue dans le commerce en utilisant les instructions du fabricant. Cependant dans le pays, il n'y a aucun contrôle de qualité de ces produits avec le risque d'une sous-estimation ou sur estimation du chlore. Ce qui rendrait inefficace la désinfection des eaux avec ces produits. $\mathrm{Ce}$ travail a été entrepris afin d'évaluer l'efficacité des eaux de javel vendues dans la ville de Lomé sur des germes isolés des eaux de boisson. 


\section{MATÉRIEL ET MÉTHODES}

Échantillonnage : Les échantillons d'eau de javel vendue dans la ville de Lomé, ont été sélectionnés de façon aléatoire et codés javel 1 à 7 . Les javels de 1 à 6 étaient sous la forme liquide tandis que la javel 7 était sous la forme de poudre. Qu'il s'agisse de la forme liquide ou en poudre, l'échantillonnage a consister à prélever au moins cinq unités de chaque marque dans leurs emballages originales.

Les échantillons d'eau analysés ont été constitués de :

- Une eau de ruissellement prélevée à Dzéméré dans la préfecture de la Kozah, codée $\mathrm{Er}$ D Ko,

- Une eau de pluie prélevée à Dzéméré dans la préfecture de la Kozah, codée Ep D Ko,

- Une eau prélevée d'une retenue de surface à Klévé dans la préfecture du Golfe, codée Ert K Go,

- Une eau de puits prélevée à Kuepédo dans la préfecture du Golfe, codée Ep Ku Go,

- $\quad$ Une eau de Forage sis à Adidogomé dans la préfecture du Golfe, codée Ef A Go,

- Une eau de rivière prélevée à Nangbéto dans la préfecture d'Est- mono, codée Eri N E-M.

L'échantillonnage de ces eaux s'est faitfaits dans des flacons stériles de $100 \mathrm{ml}$. Le transport des échantillons au laboratoire s'est fait dans des glaciaires munis d'éléments réfrigérants pour les maintenir à basse température afin de bloquer la multiplication des germes potentiels qu'ils contiennent. Les analyses microbiologiques ont été réalisées au Laboratoire de Microbiologie et de Contrôle de Qualité des Denrées Alimentaires de l'École Supérieure des Techniques Biologiques et Alimentaires (ESTBA) de I'Université de Lomé et les analyses chimiques ont été effectuées au Laboratoire de Chimie de l'ESTBA.

Analyse microbiologiques des eaux : La méthode de routine de l'Association Française de Normalisation (AFNOR) a été utilisée pour analyser les échantillons d'eau. Chaque échantillon d'eau a été dilué à $10^{-1}$ et à $10^{-2}$. Pour tous les germes recherchés et ou dénombrés, c'est l'ensemencement dans la masse qui est utilisé. II consiste à prélever $1 \mathrm{ml}$ de la solution mère ou de ses dilutions, dans une boîte de Pétri stérile et à le couvrir du milieu gélosé spécifique de chaque germe. Après solidification chaque boîte est incubée à la température spécifique au germe recherché. Les germes recherchés ont été ceux recommandés par les critères UE (Union Européenne, 2007) relatifs à l'eau de boisson. II s'est agi de la flore aérobie mésophile totale, des coliformes totaux, leset des coliformes thermotolérants, de Escherichia coli, des streptocoques fécaux, des anaérobies sulfitoréducteurs et des salmonelles. Les milieux de culture et les conditions d'incubations sont résumés dans le tableau 1.

Tableau 1 : Milieux culture et conditions d'incubation des germes recherchés dans les échantillons d'eaux

\begin{tabular}{|l|c|c|c|}
\hline Germes recherchés & Milieux de culture & $\begin{array}{c}\text { Conditions de } \\
\text { culture }\end{array}$ & $\begin{array}{c}\text { Colonies } \\
\text { caractéristiques }\end{array}$ \\
\hline $\begin{array}{l}\text { Flore aérobie mésophile } \\
\text { totale }\end{array}$ & Plate Count Agar & $30^{\circ} \mathrm{C}, 24$ à $72 \mathrm{~h}$ & $\begin{array}{c}\text { Toutes les colonies ayant } \\
\text { cultivé sur la boîte }\end{array}$ \\
\hline Coliformes totaux & Violet Red Bile Lactose & $30^{\circ} \mathrm{C}, 24 \mathrm{~h}$ & $\begin{array}{c}\text { Colonies rouges violacees } \\
\text { de diametre } \geq 0,5 \mathrm{~mm}\end{array}$ \\
\hline $\begin{array}{l}\text { Coliformes } \\
\text { thermotolérants }\end{array}$ & Violet Red Bile Lactose & $44^{\circ} \mathrm{C}, 24 \mathrm{~h}$ & $\begin{array}{c}\text { Colonies rouges violacees } \\
\text { de diametre } \geq 0,5 \mathrm{~mm}\end{array}$ \\
\hline Escherichia coli & Rapido coli & $44^{\circ} \mathrm{C}, 24 \mathrm{~h}$ & Colonies violacées \\
\hline $\begin{array}{l}\text { Anaérobie Sulfito- } \\
\text { réducteurs }\end{array}$ & $\begin{array}{c}\text { Tryptone Sulfite } \\
\text { Néomycine }\end{array}$ & $44^{\circ} \mathrm{C}, 24$ à $48 \mathrm{~h}$ & Colonies noires en tube \\
\hline Streptocoques fécaux & $\begin{array}{c}\text { Slanetz et Barthley } \\
\text { Ealmonelles }\end{array}$ & $37^{\circ} \mathrm{C}, 24 \mathrm{~h}$ & $\begin{array}{c}\text { Colonies violacées brunes } \\
\text { avec ou sans halo blanche }\end{array}$ \\
\hline EPT/Rappaport/SS & $37^{\circ} \mathrm{C}, 24 \mathrm{~h} / \mathrm{milieu}$ & $\begin{array}{c}\text { Colonies noires ou } \\
\text { incolores }\end{array}$ \\
\hline
\end{tabular}


Évaluation des propriétés antimicrobiennes des échantillons d'eau de javel : L'évaluation des propriétés antimicrobiennes des échantillons d'eau de javel a été réalisée avec une souche de référence d'Escherichia coli CIP 105182 et des souches isolées des échantillons d'eau analysés. II s'est agi de deux souches de la flore aérobie totale (une souche bacille Gram+ catalase+ et une souche bacille Gram+ catalase-), d'une souche de coliformes totaux, d'une souche de Streptocoques fécaux et d'une souche d'Escherichia coli isolées respectivement de l'eau de la rivière de Nangbéto, de l'eau de forage, de l'eau de puits et d'une retenu d'eau de surface. Les Concentrations Minimales Inhibitrices (CMI) et les Concentrations Minimales Bactéricides (CMB) ont été déterminées par la technique de dilution en milieu liquide avec le bouillon Muller Hinton (NCCLS, 2002). Les suspensions bactériennes ont été diluées dans du bouillon et distribuées dans des tubes contenant une gamme de concentration de javel allant de $2 \%, 4 \%, 6 \% 8 \% 10 \% 12 \%, 14 \% 16 \%$ (v/v) pour chacune des marques d'eau de javel étudiées. Les inocula tels que déterminés par le comptage des colonies à partir des tubes témoins ont été approximativement $10^{5} \mathrm{UFC} / \mathrm{ml}$ après étalement d'une aliquote $0,1 \mathrm{ml}$ de chaque tube sur la gélose Muller Hinton. Les tubes ont été incubés aux températures correspondant à chaque germe test. Les plus faibles concentrations de javel pour lesquelles il n'y pas eu de croissance visible à l'œil nu ont été considérées comme les CMI. Pour déterminer les CMB, les tubes qui n'ont pas présenté de turbidité ont servi pour ensemencer la gélose nutritive suivie d'une incubation de 24heures au bout desquelles, les concentrations pour lesquelles il n'y a pas eu de croissance ont été prises comme CMB.

Détermination du temps réactionnel : La détermination du temps réactionnel permet de connaitre le temps au bout duquel, l'eau de javel agit sur l'inoculum de départ mis dans le tube. Le germe le plus résistant (Germes totaux isolés de la rivière de Nangbéto, bacilles Gram+, Catalase +), les deux marques d'eau de javel (Javel 6 et javel

\section{RÉSULTATS}

Analyse microbiologique des échantillons d'eau : Les résultats de l'analyse microbiologique mentionnés dans le tableau 2 montrent que tous les échantillons d'eau analysés ne contenaient ni
7) les plus actives sur l'ensemble des germes et deux marques d'eau de javel les moins actives sur les germes (Javel 5 et javel 4) ont été utilisés pour ce test. Le germe le plus résistant et les marques d'eau de javel utilisés dans ce test sont obtenus à partir des résultats de l'évaluation des propriétés antimicrobiennes des échantillons d'eau de javel effectuée précédemment. Le test a consisté à mettre en contact $100 \mu \mathrm{l}$ de suspension du germe avec $5 \mathrm{ml}$ de javel à une concentration finale correspondant à la $\mathrm{CMB}$, le tout incubé à $30^{\circ} \mathrm{C}$. Des ensemencements successifs des aliquotes de $10 \mu \mathrm{l}$ de cette subculture sont effectués à $5,10,30$, $60,120,180,240,300,360,420$ minutes tel que décrit par Karou et al. (2006). Les milieux ainsi ensemencés sont incubés comme précédemment et les colonies dénombrées après 24 heures.

Détermination du chlore actif dans les échantillons d'eau de javel étudiés : Le chlore actif ou degré chlorométrique a été déterminé par iodométrie. Pour ce faire, $1 \mathrm{ml}$ de l'eau de javel additionné de $10 \mathrm{ml}$ d'iodure de potassium à $50 \mathrm{~g} / \mathrm{l}$, et $5 \mathrm{ml}$ d'acide acétique dans de $100 \mathrm{ml}$ d'eau distillée est titrée par une solution de thiosulfate de sodium de $0,1 \mathrm{~N}$. Au point d'équivalence (virage de la solution en jaune), quelques gouttes d'empois d'amidon ont été ajoutées et le dosage s'est poursuit jusqu'à la disparition de la coloration bleue. Pour la javel vendue sous la forme de poudre, la solution de travail a été obtenue en dissolvant $5 \mathrm{~g}$ dans $100 \mathrm{ml}$ d'eau distillée. La solution ainsi obtenue est laissée au repos pendant 24 heures avant la manipulation. Soit $\mathrm{V}$ le volume en millilitres de thiosulfate utilisé pour le dosage et $\mathrm{k}$ le facteur de dilution de la javel.

La teneur, en grammes par litre, en chlore actif est donnée par l'expression

\section{0,355 k.V}

La teneur en chlore actif exprimée en degrés chlorométriques est donnée par l'expression (Rodier, 1996) : $(0,355 \mathrm{k} . \mathrm{V}) / 3,17$

les salmonelles, ni les germes aérobies sulfitoréducteurs. Ils étaient par contre contaminés par les germes totaux, les coliformes totaux et les coliformes thermotolérants sauf, l'échantillon d'eau 
de pluie prélevée à Djéméré qui ne renfermait pas de coliformes. De même l'eau de forage ne contenait pas de coliformes thermotolérants. Tous les échantillons analysés étaient donc de qualités hygiéniques non satisfaisantes par rapport aux germes totaux et aux coliformes thermotolérants à l'exception de l'eau de pluie prélevée à Djéméré et l'eau de forage prélevé à Adidogomé. Par rapport à Escherichia coli, les échantillons d'eau de la rivière de Nangbéto, de la retenue d'eau de surface de Klévé et de l'eau de ruissellement de Djéméré étaient de qualité non satisfaisante. Par rapport aux streptocoques fécaux, les échantillons d'eau de la retenue de surface Klévé, de puits Kuepédo et l'eau de rivière de Nangbéto étaient de qualité non satisfaisante; Les six échantillons d'eau analysés étaient donc de qualités hygiéniques non satisfaisantes par rapport aux critères UE (2007) relatifs aux eaux de boissons.

Tableau 2 : Germes dénombrés dans les échantillons d'eau analysés

\begin{tabular}{|l|l|l|l|l|l|l|l|}
\hline \multirow{2}{*}{ Échantillons d'eau } & \multicolumn{6}{l|}{ Nombre de germes (UFC/ml d'eau) } \\
\cline { 2 - 8 } & GT & CT & CTH & EC & ASR & SF & Sal \\
\hline Er D Ko & 9100 & 100 & 30 & 2 & 0 & 0 & 0 \\
\hline Ep D Ko & 870 & 0 & 0 & 0 & 0 & 0 & 0 \\
\hline Ert K Go & 17000 & 3120 & 80 & 2 & 0 & 22 & 0 \\
\hline Ep Ku Go & 4000 & 740 & 50 & 0 & 0 & 80 & 0 \\
\hline Ef A Go & 20100 & 40 & 0 & 0 & 0 & 0 & 0 \\
\hline Eri N E-M & 2690 & 113 & 20 & 8 & 0 & 12 & 0 \\
\hline Critères UE, (2007) & $\mathbf{1 0 0 / m l}$ & $\mathbf{0 / 1 0 0} \mathbf{m l}$ & $\mathbf{0 / 1 0 0 m l}$ & $\mathbf{0 / 1 0 0 m l}$ & $\mathbf{2 / 2 0 m l}$ & $\mathbf{0 / 1 0 0 m l}$ & Absent/225ml \\
\hline
\end{tabular}

GT : germes totaux, CT : coliformes totaux, CTH : coliformes thermotolérants, EC : Escherichia coli, ASR : anaérobies sulfitoréducteurs, SF : streptocoques fécaux, Sal : salmonelles. Er D Ko : eau de ruissellement de Dzéméré, préfecture de la Kozah ; Ep D Ko : eau de pluie de Dzéméré, préfecture de la Kozah ; Ert K Go : eau de retenue de surface Klévé, préfecture du Golfe ; Ep Ku Go: eau de puits Kuepédo, préfecture du Golfe; Ef A Go : eau de Forage Adidogomé, préfecture du Golfe ; Eri N E-M : eau de rivière Nangbéto, préfecture d'Est- mono.

Détermination du chlore actif dans les échantillons d'eau de Javel étudiés : Les résultats de la détermination du chlore actif ou degré chlorométrique des échantillons d'eau de Javel étudiés sont consignés dans le tableau 3. L'analyse des résultats révèle que pour tous les échantillons d'eau de javel analysés, les degrés chlorométriques obtenus ne correspondaient pas à ceux mentionnés sur les emballages. Les emballages sur lesquels étaient mentionnés $8^{\circ} \mathrm{chl}$ se retrouvaient autour de $5^{\circ} \mathrm{chl}$ : cas des marques Javel 4 et Javel 5 . Concernant la javel 1 , le degré chlorométrique obtenu après dosage a été de $8,79^{\circ} \mathrm{chl}$ alors qu'il est mentionné $12^{\circ} \mathrm{chl}$ sur l'emballage. Pour toutes les marques d'eau de Javel analysées, seule la javel 2 a présenté un degré chlorométrique sensiblement égal à celui mentionné sur l'emballage (sur l'emballage $8^{\circ} \mathrm{chl}$ et après analyse $7,79^{\circ} \mathrm{chl}$ ).

Tableau 3 : Degré chlorométrique des échantillons d'eau de Javel

\begin{tabular}{|l|c|c|}
\hline Échantillons & $\begin{array}{c}\text { Degré chlorométrique inscrit sur } \\
\text { l'emballage, }\end{array}$ & $\begin{array}{c}\text { degré chlorométrique obtenu après } \\
\text { dosage du chlore actif }\end{array}$ \\
\hline Javel 1 & $12^{\circ} \mathrm{chl}$ & $8,79^{\circ} \mathrm{chl}$ \\
\hline Javel 2 & $8^{\circ} \mathrm{chl}$ & $7,79^{\circ} \mathrm{chl}$ \\
\hline Javel 3 & $36^{\circ} \mathrm{chl}$ & $15,454^{\circ} \mathrm{chl}$ \\
\hline Javel 4 & $8^{\circ} \mathrm{chl}$ & $5,543^{\circ} \mathrm{chl}$ \\
\hline Javel 5 & $8^{\circ} \mathrm{chl}$ & $5,77^{\circ} \mathrm{chl}$ \\
\hline Javel 6 & $36^{\circ} \mathrm{chl}$ & $0,0335^{\circ} \mathrm{chl}$ \\
\hline Javel 7 & non inscrit sur l'emballage & $9,85^{\circ} \mathrm{chl}$ \\
\hline
\end{tabular}


Évaluation des propriétés antimicrobiennes des échantillons d'eau de javel étudiés : Les résultats de l'évaluation des propriétés antimicrobiennes de chaque marque d'eau de javel sont exprimés en termes de concentration minimale inhibitrice (CMI) et de concentration minimale bactéricide (CMB) puis consignés dans le tableau 4. Les résultats montrent que les $\mathrm{CMI}$ obtenues pour les sept types d'eau de javel sont comprises entre 2 (666mg) et 12,5\% (4166mg) alors que les CMB sont comprises entre 2,5 (883mg) et $16,6 \%$ (5533mg). En considérant les résultats obtenus par germe, les $\mathrm{CMI}$ sont comprises entre 2 et $5 \%$ et les CMB entre 2,5 et $6,25 \%$ pour les Streptocoques fécaux, les coliformes totaux et les germes totaux isolés de l'eau de forage. Concernant les germes $E$. coli CIP105 182 et $E$. coli isolée de l'eau de puits, les CMI ont varié entre 2 et $6,25 \%$ alors que les CMB ont varié entre 2,5 et $8,33 \%$. Les germes totaux isolés de la rivière de Nangbéto ont eu des $\mathrm{CMI}$ entre 5 et $12,5 \%$ tandis que les CMB ont été de 6,25 à $16,6 \%$. Ce qui justifierait le fait que les germes les plus résistants parmi les germes testés soient les germes totaux isolés de l'eau de Nangbéto (Bacilles Gram+, Catalase +) car donnant les plus grandes valeurs de CMl et CMB avec de plus grandes valeurs de chlore actif. En prenant les valeurs des CMI et CMB des diverses marques d'eau de javel étudiées par rapport aux germes, les plus petites valeurs se retrouvent au niveau de l'eau de Javel « javel 6 » (CMl allant de 2 à $6,25 \%$ et CMB allant de 2,5 à $8,33 \%$ ) suivi de la Javel 7 (CMl allant de 2 à $5 \%$ et CMB allant de 2,5 à $6,25 \%$ ). Ceci permet de déduire que l'eau de javel la plus efficace est la marque d'eau de javel 6 . Un test de corrélation a été réalisé entre les degrés chlorométriques obtenus après dosage des différents types d'eau de javel étudiés à l'exception de la javel 7 et les degrés chlorométriques mentionnés sur leur emballage. En déterminant cette corrélation avec la javel 6 , on obtient une valeur de 0,0987 tandis qu'en l'excluant de la série, on obtient une valeur de 0,998. II ressort de ce fait que pour les 5 types d'eau de javel (les javels 1, 2, 3, 4 et 5) la différence entre les degrés chlorométriques obtenus au dosage et ceux inscrits sur l'emballage du produit est liée au même facteur qui pourrait être la surestimation par le fabriquant ou l'altération du chlore. Par contre, la différence entre le degré chlorométrique obtenu au dosage de la javel 6 et celui inscrit sur son emballage est liée à un autre facteur. Des tests de corrélation ont été effectués entre les degrés chlorométriques des échantillons d'eau de javel obtenus après leur dosage et leurs $\mathrm{CMl}$ par rapport aux germes d'une part et leurs CMB d'autre part. Les résultats de ces tests sont consignés dans le tableau 5. 
Djeri et al. J. Appl. Biosci. 2016 Évaluation des propriétés antimicrobiennes des javels vendues à Lomé sur quelques germes isolés de l'eau de consommation

Tableau 4 : valeurs des CMl et CMB en \% (v/v)

\begin{tabular}{|c|c|c|c|c|c|c|c|c|c|c|c|c|c|c|}
\hline \multirow[t]{2}{*}{ Germes testés } & \multicolumn{2}{|c|}{ Javel 1} & \multicolumn{2}{|c|}{ Javel 2} & \multicolumn{2}{|c|}{ Javel 3} & \multicolumn{2}{|c|}{ Javel 4} & \multicolumn{2}{|c|}{ Javel 5} & \multicolumn{2}{|c|}{ Javel 6} & \multicolumn{2}{|c|}{ Javel 7} \\
\hline & CMI & CMB & CMI & CMB & CMI & CMB & CMI & CMB & CMI & CMB & CMI & CMB & CMI & CMB \\
\hline $\begin{array}{l}\text { Streptocoques } \\
\text { fécaux }\end{array}$ & 5 & 6,25 & 5 & 6,25 & 5 & 6,25 & 4 & 5 & 4 & 5 & 2 & 2,50 & 5 & 6,25 \\
\hline $\begin{array}{l}\text { Coliformes } \\
\text { Totaux. }\end{array}$ & 5 & 6,25 & 5 & 6,25 & 5 & 6,25 & 5 & 6,25 & 5 & 6,25 & 2 & 2,50 & 2,85 & 4 \\
\hline E. coli & 2,50 & 2,85 & 5 & 6,25 & 5 & 6,25 & 6,25 & 8,33 & 6,25 & 8,33 & 2 & 2,50 & 2,50 & 2,85 \\
\hline $\begin{array}{l}\text { Bacilles Gram + } \\
\text { Catalase + }\end{array}$ & 5 & 6,25 & 1,25 & 1,66 & 6,25 & 8,33 & 1,25 & 1,66 & 1,25 & 1,66 & 6,25 & 8,33 & 5 & 6,25 \\
\hline $\begin{array}{l}\text { Bacilles Gram + } \\
\text { Catalase - }\end{array}$ & 5 & 6,25 & 5 & 6,25 & 5 & 6,25 & 5 & 6,25 & 5 & 6,25 & 2 & 2,50 & 2,85 & 4 \\
\hline $\begin{array}{l}\text { E. coli CIP } \\
105182\end{array}$ & 5 & 6,25 & 5 & 6,25 & 2,50 & 2,85 & 6,25 & 8,33 & 4 & 5 & 2 & 2,50 & 2 & 2,50 \\
\hline
\end{tabular}

Tableau 5 : Résultats des tests de corrélation entre le degré chlorométrique des échantillons

\begin{tabular}{|l|l|l|}
\hline Germes testés & Coefficient de corrélation \\
\cline { 2 - 3 } & ${ }^{\circ}$ Chl/CMI & ${ }^{\circ}$ Chl/CMB \\
\hline Streptocoques fécaux & 0,846 & 0,846 \\
\hline Coliformes totaux & 0,494 & 0,541 \\
\hline E. coli & 0,178 & 0,118 \\
\hline Bacille Gram+ Catalase + & $-0,262$ & $-0,265$ \\
\hline Bacille Gram+- Catalase- & 0,499 & 0,541 \\
\hline E. coli CIP 105 182 & $-0,093$ & $-0,135$ \\
\hline
\end{tabular}


Les résultats de ces tests révèlent que les valeurs des corrélations entre les degrés chlorométriques des échantillons d'eau de javel et les CMI d'une part sont très proches des valeurs des corrélations entre les degrés chlorométriques et les CMB d'autre part. Cette remarque permet d'affirmer que l'action inhibitrice de chaque échantillon d'eau de javel étudiés est presque la même que son action bactéricide. Par ailleurs, il existe une corrélation positive à $66,66 \%$ entre le degré en chlore actif des solutions d'eau de javel et leurs actions inhibitrices et bactéricides sur les germes. Ces germes sont donc sensibles à la quantité de chlore présente dans les différents types d'eau de javel étudiés. II s'agit dans un ordre décroissant de sensibilité des germes : streptocoques fécaux, Bacille Gram+ catalase-, Coliformes totaux, E. coli. Par contre, les germes Bacilles Gram+ Catalase + et $E$. coli CIP 105182 (soient 33,33\% des germes) donnent des valeurs négatives de ces corrélations.
Ces valeurs négatives pourraient montrer une possible résistance de ces deux germes en questions par rapport au chlore, soit une corrélation inverse (l'action bactéricide diminue quand la concentration en chlore actif des solutions augmente).

Détermination du temps réactionnel : La détermination du temps réactionnel à été faite avec la souche de germes totaux Bacille à Gram+ catalase+ et les marques d'eau de javel 6 et 7. Les résultats obtenus révèlent qu'aucune marque d'eau de javel n'agit sur la totalité des germes totaux Bacilles Gram+ catalase+ in-vitro avant 2 heures. Dans le cadre de cette étude, les eaux de javel " efficaces " sont celles qui ont inhibé presque la totalité des germes à 3 heures alors que les eaux de javel moins efficaces sont celles qui ont agi sur la totalité des germes autour de 6 heures de temps (figure 1).

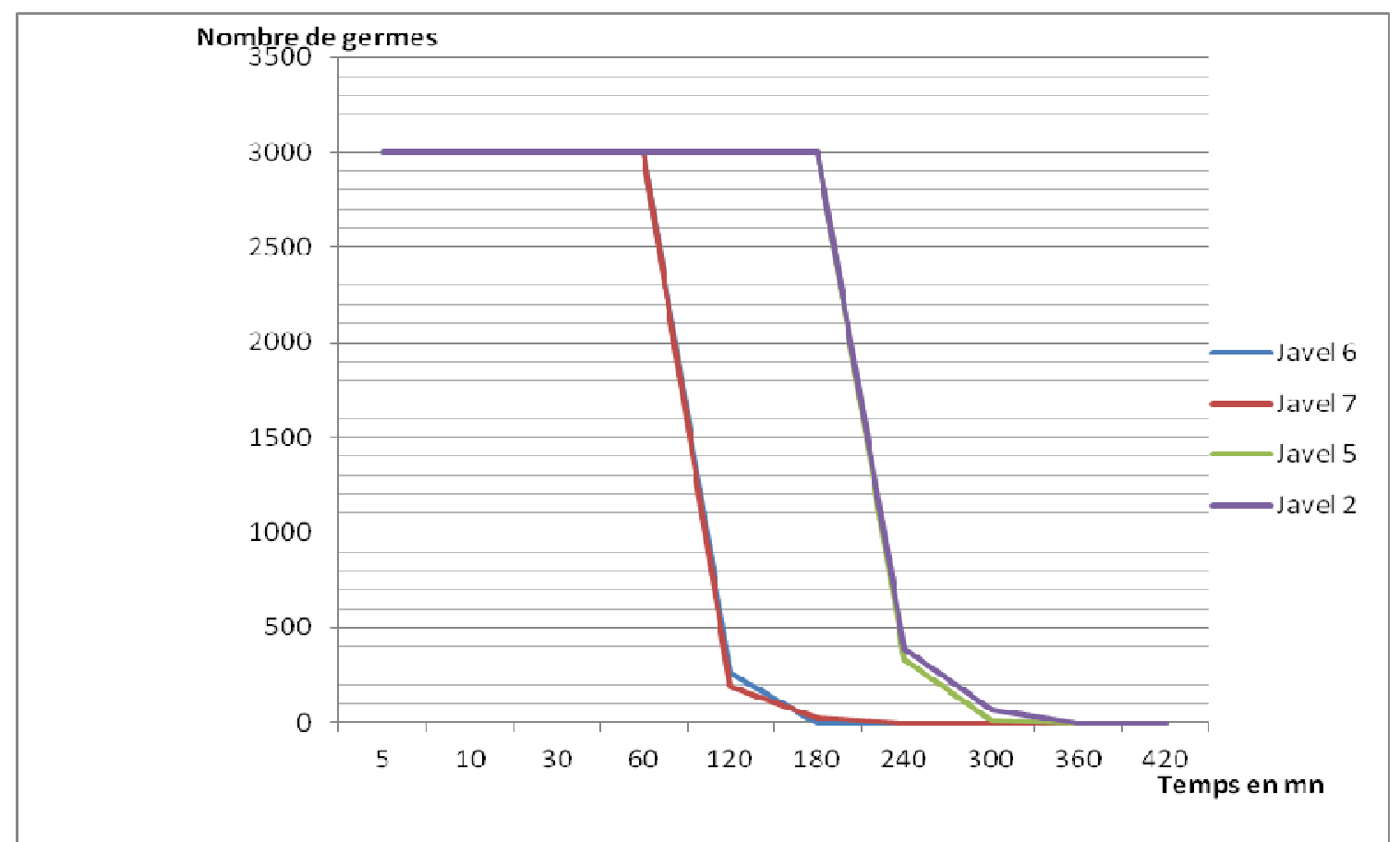

Figure 1_: Evolution de la charge microbienne en UFC en fonction du temps

(on considère qu'il y a envahissement lorsque la charge microbienne est supérieure à 3000 UFC).

\section{DISCUSSION}

La présente étude a eu pour objectif l'évaluation de l'efficacité de différentes marques d'eau de javel vendues dans le commerce dans la ville de Lomé au Togo. II faut noter que ces javels sont le plus souvent employées dans les opérations de désinfections de l'eau de boisson et des légumes en usage domestique. Dans une première partie il s'est agi d'analyser certains échantillons d'eau que les populations utilisent comme eau de boisson. Les résultats de cette analyse ont montré que 
toutes les eaux analysées étaient exemptes de Salmonelles et de bactéries anaérobies sulfitoréductrices. Cependant toutes ces eaux contenaient une flore aérobie totale et des coliformes totaux, de charge élevée par rapport à celle recommandée par. Suivant les critères UE (2007) pour les eaux de boissons.. La nonconformité des eaux de boisson dans les pays en voie de développement a été soulignée par plusieurs auteurs qui se sont intéressés à la qualité microbiologique des eaux de consommation dans les cités africaines (Kouadio et al., 1998 ; Degbey et al., 2008 ; Haoussi et al., 2011, 2013). Pour ce qui est de la ville de Lomé, les travaux de Kimassoum et al. (2011) ont montré que les eaux de la "Togolaise des Eaux (TdE)», principal réseau de distribution urbaine du pays, étaient contaminées. II faut noter que les eaux analysées dans cette étude sont des eaux de forage, des puits ou de retenues qui n'ont subi aucun traitement contrairement aux eaux de la TdE qui sont traitées. Ce résultat n'est donc pas surprenant d'autant plus que Tampo et al. (2014) qui ont travaillé sur les eaux de puits de la ville de Lomé ont trouvé que toutes ces eaux étaient non conformes aux critères UE, (2007). Vu la nonconformité de ces eaux de forage et de puits, certains ménages au Togo pratiquent une désinfection empirique de ces eaux avec la javel en se référant aux prescriptions sur l'emballage avant la consommation. Les tests antimicrobiens effectués avec les eaux de javel nous ont montré que toutes ces échantillons inhibent à des degrés divers les germes testés. Les concentrations minimales bactéricides les plus petites ont été de $2,5 \%$. Ces résultats, démontrent une efficacité certaine des eaux de javel vendues à Lomé. Cependant un problème de conformité entre la valeur du degré chlorométrique mentionné sur l'emballage et le degré chlorométrique réel de ces produits se pose, le chlore étant le principe actif des eaux de javel. Ce constat nous a incités à déterminer expérimentalement les degrés chlorométriques de ces marques d'eau de javel. Les résultats ont montré que seule une marque de javel avait un degré chlorométrique expérimental qui s'approchait de celui mentionné sur l'emballage. Pour les autres marques, le degré chlorométrique expérimental était largement en dessous de la valeur mentionnée avec des écarts pouvant faire plus du double de la valeur expérimentale. II est évident que l'iodométrie utilisée dans la présente étude présente des biais mais l'écart observé avec certaines marques devrait attirer l'attention. Cependant, cette différence notable entre les concentrations en chlore actif obtenues par dosage et celles mentionnées sur l'emballage des échantillons d'eau de javel peut être liée aux facteurs de décomposition de l'eau de javel que sont la chaleur, la lumière et en particulier les rayonnements UV (Durliat et al., 1997). Par ailleurs, l'air participe également à la décomposition naturelle de l'eau de javel et cette décomposition dépend de la température et de la concentration en chlore de la solution (Festy et al., 2003 ). Cette différence peut être due aussi à une mauvaise dilution des eaux de javel par les fabricants ou dans la chaîne de vente ou encore à la durée de conservation. En effet, les travaux de Ballereau et al. (1997) ont dévoilé plusieurs facteurs accélérant la dégradation de l'hypochlorite de sodium. L'exposition à la lumière est un facteur fondamental. La diminution du titre en chlore entre $\mathrm{J} 0$ et J35 est approximativement de $20 \%$ lorsque la solution est conservée dans un flacon de verre alors qu'elle n'est que de $5 \%$ si le même flacon est conservé à l'abri de la lumière. L'air est également un facteur de réduction très important de la teneur en chlore, en particulier si la solution est exposée à la lumière, avec une action synergique de ces deux facteurs. La teneur en chlore diminue de moitié entre $\mathrm{J} 0$ et $\mathrm{J} 35$, lorsque la solution est conservée dans un flacon en verre blanc ouvert et exposé à la lumière. D'après la société des produits chimiques Harbonnières basée en France, le chlore se décompose de façon naturelle sous l'effet de la chaleur et de la concentration. Ainsi, une augmentation de $5^{\circ} \mathrm{C}$ accélère la vitesse de réaction de décomposition du chlore par 2.La grande efficacité des javels 6 et 7 sur les germes étudiés pourrait s'expliquer par le fait que ces eaux de javel ont été frelatée en remplaçant le chlore par un autre désinfectant puissant et moussant. $\mathrm{Ce}$ composé de remplacement peut être de la famille des ammoniums quaternaires qui sont décrits par Gérard (1981) comme des liquides sirupeux. Les solutions sont très stables et ne sont altérées ni par le gel ni par la chaleur, elles peuvent donc être conservées à la température ambiante. Elles sont très solubles dans l'eau quel que soit le $\mathrm{pH}$. 


\section{CONCLUSION}

Au terme de cette étude, il en ressort que sur le marché togolais il existe des marques d'eau de javel de divers degrés d'efficacité. Le dosage du degré chlorométrique des différentes marques étudiées a révélé que les degrés chlorométriques

\section{REFERENCES}

Ahoussi KE, Oga YMS, Koffi YB, Kouassi AM, Soro N, Biemi J, 2011. Caractérisation hydrogéochimique et microbiologique des ressources en eau du site d'un Centre d'Enfouissement Technique (CET) de Côte d'Ivoire : cas du CET de Kossihouen dans le District d'Abidjan (Côte d'Ivoire). International Journal of Biological and Chemical Sciences; 5(5):2114-32. Available from: http://www.ajol.info/index.php/iibcs/article/ view/77205

Ahoussi KE, Koffi YB, Kouassi AM, Soro G, Biemi $J$, 2013. Étude hydrochimique et microbiologique des eaux de source de l'ouest montagneux de la Côte d'Ivoire: Cas du village de Mangouin-Yrongouin (sous-préfecture de Biankouman). Journal of Applied Biosciences; 63(1):4703-19. Available from: http://www.ajol.info/index.php/jab/article/vi ew/87245

Ballereau F, Merville C, Lafleuriel MT, Schrive I, 1997. Stabilité et efficacité antimicrobienne de l'eau de Javel en milieu hospitalier tropical. Bulletin de la Société de pathologie exotique ; 90(3): 192-5. Available from : http://www.pathexo.fr/documents/articlesbull/T90-3-1800.pdf

Bawa LM, Tchakala I, Djanéyé-Bouindjou G, 2008. Détermination de la demande en chlore des eaux de puits et de forages d'un quartier périurbain de la ville de Lomé : incidence sur la désinfection Assessment of chlorine demand of water. Available from: http://www.samptf.com/pdf/vol7 2 4.pdf\% 20M\%20Bawa \%20et\%20al.pdf

Becerra S, Roussary A, 2008. Gérer la vulnérabilité de l'eau potable: une action publique désengagée ? Natures Sciences Sociétés ; 16(3):220-31. Available from : réels des échantillons d'eau de javel étudiés sont inférieurs à ceux mentionnés sur les emballages. II est donc important d'effectuer des contrôles périodiques de ces produits pour s'assurer de l'efficacité des opérations de désinfection.

http://www.cairn.info/resume.php?ID_ART ICLE=NSS $163 \quad 0220$

Dégbey $C$, Makoutode $M$, Ouendo E-M, Fayomi $B$, De Brouwer C, 2008. La qualité de l'eau de puits dans la commune d'AbomeyCalavi au Bénin. Environnement, Risques \& Santé ; 7(4):279-83. Available from: http://www.jle.com/fr/revues/ers/edocs/la_qualite_de_leau_de_puits_dans_I a_commune_dabomey_calavi_au_benin_ 278249/article.phtml

Degbey C, Makoutode M, Agueh V, Dramaix M, de Brouwer $C, 2011$. Facteurs associés à la qualité de l'eau de puits et prévalence des maladies hydriques dans la commune d'Abomey-Calavi (Bénin). Cahiers d'études et de recherches francophones/Santé ; $\quad$ 21(1):47-55. Available from : http://www.jle.com/fr/revues/san/edocs/facteurs_associes_a_la_qualite_de_I eau de puits et prevalence des maladi es_hydriques_dans_la_commune_dabom ey calavi benin 289183/article.phtml

Durliat G, Vignes J-L, Joffin JN, 1997. L'eau de Javel: sa chimie et son action biochimique. Bulletin de l'union des physiciens; 91:451-71. Available from: http://www.ch4service.fr/science/analyses/ eau-de-javel.pdf

Festy $B$, Hartemann $P$, Ledrans $M$, Levallois $P$, Payment $P$, Tricard D, 2003. Qualité de l'eau. Gerin M, Gosselin P, Cordier S, Viau C, Quenel P, Dewailly E, Environnement et Santé Publique Fondements et pratiques Ed Tec \& Doc Paris; Available from : http://www.dsest.umontreal.ca/documents 119Chap13.pdf

Gerard JP, 1981. Les ammoniums quaternaires en thérapeutique piscicole. Bulletin Français de Pisciculture; ;280):128-30. Available from : $\quad$ http://www.kmae- 
journal.org/articles/kmae/pdf/1981/01/kma e198128010.pdf

Karou D, Savadogo A, Canini A, Yameogo S, Montesano C, Simpore J, V Colizzi, et AS Traore, 2005. Antibacterial activity of alkaloids from Sida acuta. African Journal of Biotechnology; 4(12). Available from: http://www.ajol.info/index.php/ajb/article/vi ew/71463

Kimassoum D, Tidjani A, Doutoum A, Ameyapoh Y, Soncy K, Dossou K, ANANI K. T.; SOUZA C.., 2011. Évaluation de la qualité hygiénique de l'eau de robinet produite par la Société Togolaise des Eaux (TdE) : cas de neuf quartiers de la commune de Lomé (Togo). MHA ; 23(68) :51-4. Available from: http://cat.inist.fr/?aModele=afficheN\&cpsid $\mathrm{t}=27392059$

Kouadio LP, Ekra NB, Atindehou E, Nanou C, Monnet D, 2008. Étude de la potabilité des eaux de boisson en sachet vendues aux abords des écoles primaires publiques d'Abidjan. Bulletin de la Société de pathologie exotique; 2:167-8. Available from : http://www.pathexo.fr/documents/articlesbull/T91-2-1766.pdf

Lesne J, 1998. Hygiène publique, microbiologie et gestion de l'eau. Bull Soc Pathol Exot; 91:438-44. Available from: http://www.pathexo.fr/documents/articlesbull/T91-5-PF13.pdf

Matthys $B$, Adiko $F A$, Cissé $G, 2006$. Le réseau social des maraîchers à Abidjan agit sur la perception des préoccupations et des risques sanitaires liés à l'eau. VertigO-la revue électronique en sciences de l'environnement;(Hors-série 3). Available from: http://vertigo.revues.org/1857

National Committee for Clinical Laboratory Standards, 2002. Reference Methods for Broth Dilution Antifungal Susceptibility Testing of Yeast: Approved Standar. National Committee for Clinical Laboratory Standards.

Poda JN, 2007. Les maladies liées à l'eau dans le bassin de la Volta: état des lieux et perspectives. Volta Basin Focal Project report; 4. Available from:
http://cpwfbfp.pbworks.com/f/BFP\%20Volt a Poda\%20(2007).pdf

Tampo L, Ayah M, Kodom T, Tchakakla I, Boguido P, Bawa L, B Djaneye 2014. Impact de la demande en chlore et de la chloration sur la désinfection des eaux de puits des quartiers de Lomé : cas des quartiers de Démakpoé et d'Agbalépédogan (Togo). Journal of Applied Biosciences; 75(1):6272-81. Available from: http://www.ajol.info/index.php/jab/article/vi ew/102661

Tessier S, 1992. Les maladies de l'enfant liées à l'eau en milieu urbain. Cahiers d'études et de recherches francophones/Santé ; 2(2) :77-84. Available from: http://www.jle.com/fr/revues/san/edocs/les_maladies_de_lenfant_liees_a_le au en milieu urbain 270552/article.phtm ! 